\title{
First Steps towards Initial Registration for Electrophysiology Procedures
}

\author{
Alexander Brost ${ }^{a}$, Felix Bourier ${ }^{b}$, Liron Yatziv $^{c}$, Martin Koch ${ }^{a}$, Joachim \\ Hornegger $^{a}$, Norbert Strobel $^{d}$ and Klaus Kurzidim ${ }^{b}$ \\ ${ }^{a}$ Pattern Recognition Lab, Friedrich-Alexander-University \\ Erlangen-Nuremberg, Erlangen, Germany \\ ${ }^{b}$ Klinik für Herzrhythmusstörungen, Krankenhaus Barmherzige Brüder, \\ Regensburg, Germany \\ ${ }^{c}$ Siemens Corporate Research, Mountain View, CA, USA \\ ${ }^{d}$ Siemens AG, Healthcare Sector, Forchheim, Germany
}

\begin{abstract}
Atrial fibrillation is the most common heart arrhythmia and a leading cause of stroke. The treatment option of choice is radio-frequency catheter ablation, which is performed in electrophysiology labs using $\mathrm{C}$-Arm $\mathrm{X}$-ray systems for navigation and guidance. The goal is to electrically isolate the pulmonary vein-left atrial junction thereby rendering myocardial fibers responsible for induction and maintenance of AF inactive. The use of overlay images for fluoroscopic guidance may improve the quality of the ablation procedure, and can reduce procedure time. Overlay images, acquired using CT, MRI, or C-arm CT, can add soft-tissue information, otherwise not visible under X-ray. MRI can be used to image a wide variety of anatomical details without ionizing radiation. In this paper, we present a method to register a 3-D MRI volume to 2-D biplane X-ray images using the coronary sinus. Current approaches require registration of the overlay images to the fluoroscopic images to be performed after the trans-septal puncture, when contast agent can be administered. We present a new approach for registration to align overlay images before the trans-septal puncture. To this end, we manually extract the coronary sinus from pre-operative MRI and register it to a multi-electorde catheter placed in the coronary sinus.
\end{abstract}

Keywords: Registration, Atrial Fibrillation, Radiofrequency Catheter Ablation, Electrophysiology

\section{MOTIVATION}

As atrial fibrillation is widely recognized as the most common heart arrhythmia and a leading cause of stroke. Its treatment is usually performed by radio-frequency catheter ablation (RFCA) performed in electrophysiology (EP) labs [1]. During these procedures, the left atrium (LA) is electrically isolated by ablating around the ostia of the attached pulmonary veins (PVs) [2]. Overlay images for fluoroscopic guidance improve the quality of the ablation, and they can reduce procedure times [3]. Intra-procedural imaging for catheter guidance is performed by C-arm X-ray systems. During such RFCA procedures, physicians regularly place one mapping catheter in the coronary sinus (CS) for pacing. Another advantage of inserting a catheter into the CS is some visual feedback about the position and orientation of the left atrium. The next and crucial step during the procedure is when the inter-atrial septum - the wall between the left and right atrium - is punctured to bring the ablation and mapping catheters into the left

Send correspondence to Alexander Brost: E-mail: Alexander.Brost@ informatik.uni-erlangen.de 


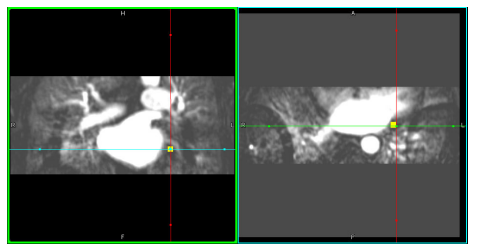

(a)

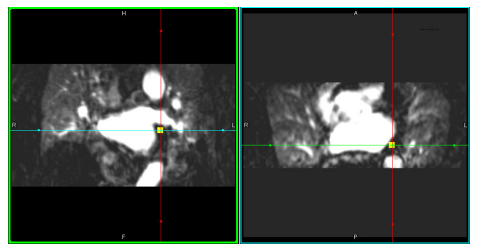

(d)

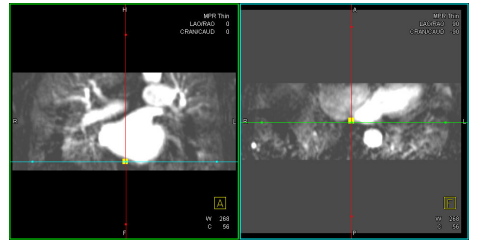

(b)

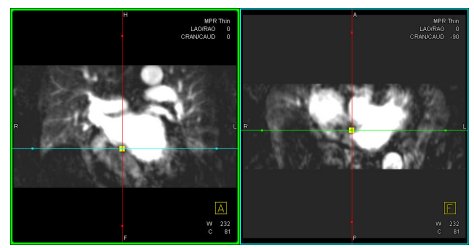

(e)

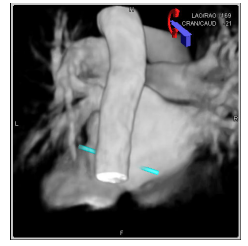

(c)

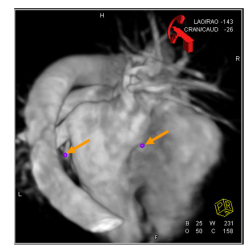

(f)

Figure 1: Two examples for coronary sinus vein localization in MRI. (a),(d) We locate the coronary sinus in the triangle of tricuspide valve, interatrial septum and inferior vena cava. (b),(e) The transition of the CS to the Great Cardiac Vein is found caudal to the left pulmonary veins. Afterwards, the MPR slices are adjusted to show both set of points. (c),(f) The extracted CS is visualized in 3-D together with the pre-operative MRI.

atrium. Visual guidance can be obtained by using trans-esophageal echo-cardiography (TEE) or intra-cardiac echo-cardiography (ICE). As soon as a catheter sheath has been inserted into the left atrium, contrast agent can be administered to verify success. In the following sections, we present a first approach to perform registration of overlay-images without requiring TEE or ICE before the trans-septal puncture.

\section{METHODS}

In this section, the extraction of the coronary sinus from 3-D MRI, the extraction of the mapping catheter in the coronary sinus and the iterative closest point algorithm are briefly explained.

\subsection{Coronary Sinus Localization in 3-D Data}

In the first step, the coronary sinus is manually extracted from the volumetric data set, in our case a MRI. The coronary sinus is located in the triangle of tricuspide valve, interatrial septum and inferior vena cava. The transition of the CS to the Great Cardiac Vein is found caudal to the left pulmonary veins. The extraction workflow is summarized in Figure 1. It is possible to extract the remaining parts of the coronary sinus from the MRI as well, but only the starting and end point $\mathbf{p}_{s}, \mathbf{p}_{e} \in \mathbb{R}^{3}$ of the $\mathrm{CS}$ are required for registration. Placing a catheter into the coronary sinus leads to a deformation of the left atrium, i.e., a linearization of the vein. This is due to the fact that the catheters still have a certain stiffness that does not completely follow the coronary sinus vein. This is also visualized in Figures 2 and 3. To deal with the deformation, the two points are used to define a line $l(\lambda)$ as

$$
l(\lambda)=\mathbf{p}_{s}+\lambda \cdot \frac{\mathbf{p}_{e}-\mathbf{p}_{s}}{\left\|\mathbf{p}_{e}-\mathbf{p}_{s}\right\|_{2}}
$$

This line is the likely position of the mapping catheter when placed in the coronary sinus. To obtain discrete samples $\mathbf{p}_{i} \in \mathbb{R}^{3}$ the line is sub-sampled into $N \in \mathbb{N}$ equally spaced segments, thus resulting to

$$
\mathbf{p}_{i}=\mathbf{p}_{s}+\frac{i}{N} \cdot \frac{\mathbf{p}_{e}-\mathbf{p}_{s}}{\left\|\mathbf{p}_{e}-\mathbf{p}_{s}\right\|_{2}}
$$

with $i \in[0, N]$. 


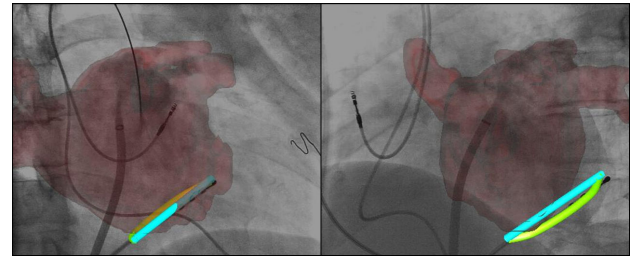

(a)

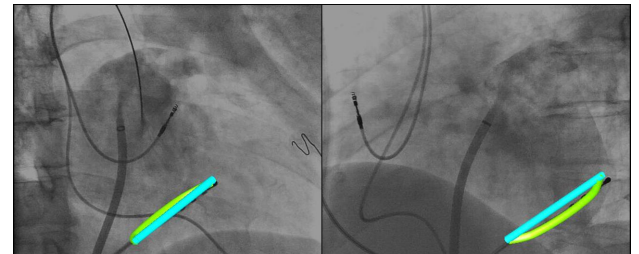

(b)

Figure 2: (a) Fluoroscopic overlay of segmented LA with assumed coronary sinus (green line). Linear coronary sinus (straight blue line) shows assumed position of CS-Catheter and is used for registration. CS-Catheter is placed in Coronary Sinus. (b) The same images as left - without overlay.

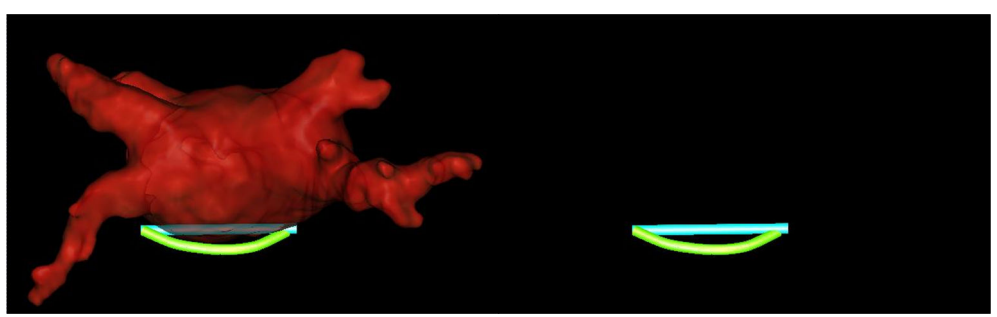

(a)

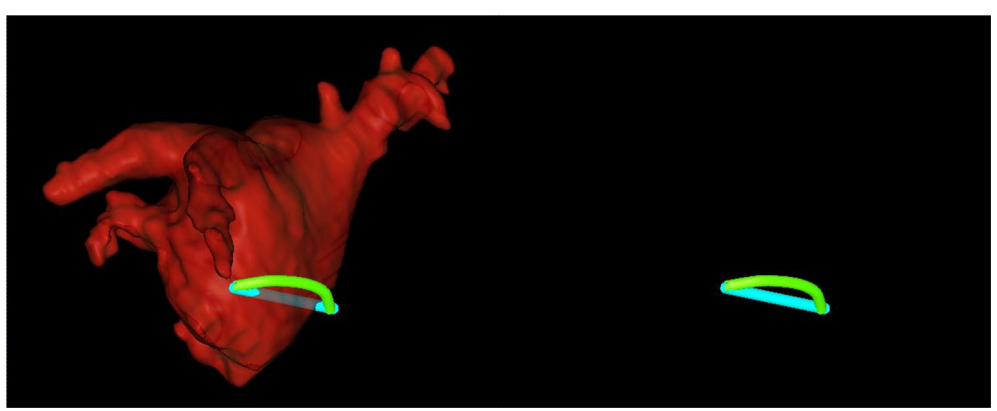

(b)

Figure 3: Segmented LA with extracted coronary sinus (green). The deflection between the green and blue lines show the deformation of the coronary sinus caused by inserted catheter.

\subsection{Extraction from X-Ray Images}

The extraction of the mapping catheter in the coronary sinus is performed by successively triangulating points from two views, see Figure 4 for an illustration. The underlying algorithm for point reconstruction from two views is described in $[4,5]$. The projection matrices for the imaging planes, plane $\mathrm{A}$ and plane $\mathrm{B}, \mathbf{P}_{A}, \mathbf{P}_{B} \in \mathbb{R}^{3 \times 4}$ are given as

$$
\begin{aligned}
& \mathbf{P}_{A}=\mathbf{K}_{A}\left[\mathbf{R}_{A} \mid \mathbf{t}_{A}\right] \\
& \mathbf{P}_{B}=\mathbf{K}_{B}\left[\mathbf{R}_{B} \mid \mathbf{t}_{B}\right]
\end{aligned}
$$

with the intrinsic camera parameters denoted as $\mathbf{K}_{A}, \mathbf{K}_{B} \in \mathbb{R}^{3 \times 3}$ and the extrinsic camera parameters comprising rotation $\mathbf{R}_{A}, \mathbf{R}_{B} \in \mathbb{R}^{3 \times 3}$ and translation $\mathbf{t}_{A}, \mathbf{t}_{B} \in \mathbb{R}^{3}$. The 2-D points of the catheter for each imaging plane are denoted as $\mathbf{v}_{A} \in \mathbb{R}^{2}$ for plane $A$ and $\mathbf{v}_{B} \in \mathbb{R}^{2}$ for 


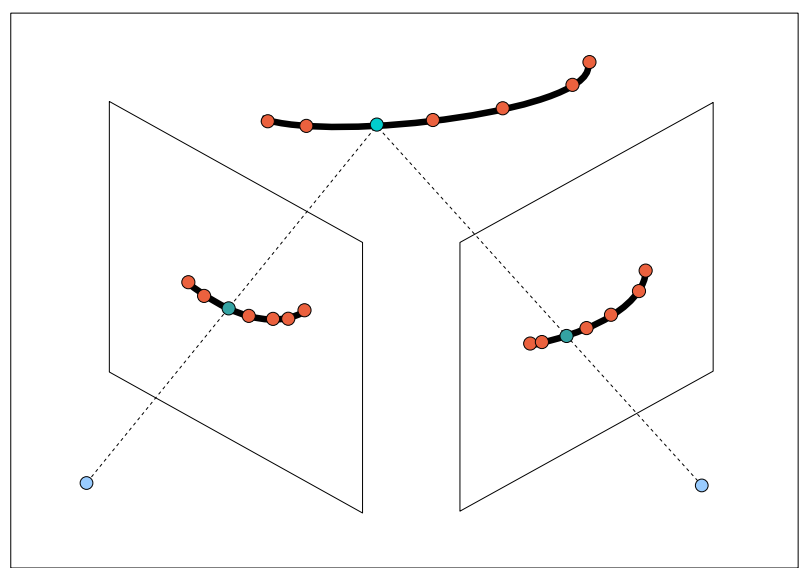

Figure 4: Successive localization by triangulation of the mapping catheter in the coronary sinus from two views.

plane B. Rewriting these points in a higher dimension as

$$
\begin{aligned}
& \mathbf{v}_{A}^{\star}=\left(\begin{array}{c}
\mathbf{v}_{A} \\
1
\end{array}\right) \\
& \mathbf{v}_{B}^{\star}=\left(\begin{array}{c}
\mathbf{v}_{B} \\
1
\end{array}\right)
\end{aligned}
$$

is used to compute two rays in 3-D space as

$$
\begin{aligned}
& \mathbf{r}_{A}(\mu)=-\mathbf{R}_{A}^{T} \mathbf{t}_{A}+\mu \mathbf{R}_{A}^{T} \mathbf{K}_{A}^{-1} \mathbf{v}_{A}^{\star} \\
& \mathbf{r}_{B}(\nu)=-\mathbf{R}_{B}^{T} \mathbf{t}_{B}+\nu \mathbf{R}_{B}^{T} \mathbf{K}_{B}^{-1} \mathbf{v}_{A}^{\star} .
\end{aligned}
$$

Intersecting these two rays, $\mathbf{r}_{A}(\mu)$ and $\mathbf{r}_{B}(\nu)$, leads to a point in 3-D which projection onto the imaging planes coincides with $\mathbf{v}_{A}$ and $\mathbf{v}_{B}$. Successive localization by triangulation of the mapping catheter in the coronary sinus from two views yields to 3-D points denoted as $\mathbf{q}_{j} \in \mathbb{R}^{3}$.

\subsection{Iterative Closest Point}

Iterative closest point (ICP) is an algorithm used to minimize the distance between two objects - represented by sets of points - based on a mean square error measure $[6,7]$. Two point sets, a moving and a fixed point set, are given as

$$
\begin{aligned}
\mathcal{P} & =\left\{\mathbf{p}_{i}\right\} \\
\mathcal{Q} & =\left\{\mathbf{q}_{j}\right\}
\end{aligned}
$$

with the points $\mathbf{p}_{i}, \mathbf{q}_{j} \in \mathbb{R}^{3}$ and its cardinalities $|\mathcal{P}|=N$ and $|\mathcal{Q}|=M$. Using these two point groups, Transformation parameters for rotation and translation can be estimated using a mean square cost function $d(\cdot)$. We considered only translation $\mathbf{t} \in \mathbb{R}^{3}$ for our approach. This leads to the following mean square cost function

$$
d(\mathbf{t})=\frac{1}{N} \sum_{i}\left\|\min _{q_{j} \in \mathcal{Q}}\left(p_{i}+t, q_{j}\right)\right\|^{2} .
$$

To find the optimal translation vector $\hat{\mathbf{t}}$, the moving point set needs to updated after each iteration $k$ by

$$
\mathcal{P}_{k+1}=\left\{\mathbf{p}_{i, k}+\mathbf{t}_{k}\right\}
$$




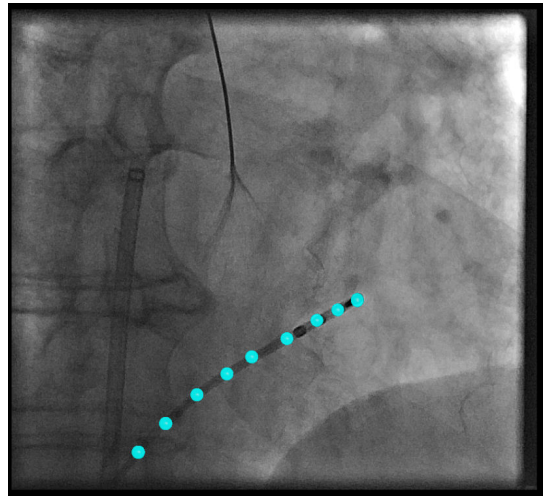

(a) Imaging Plane A

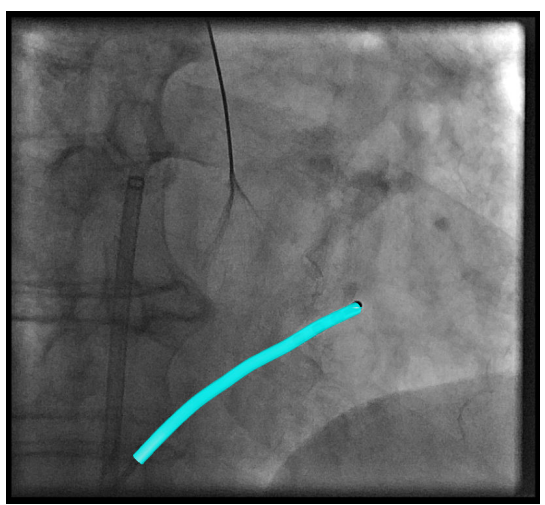

(c) Imaging Plane A

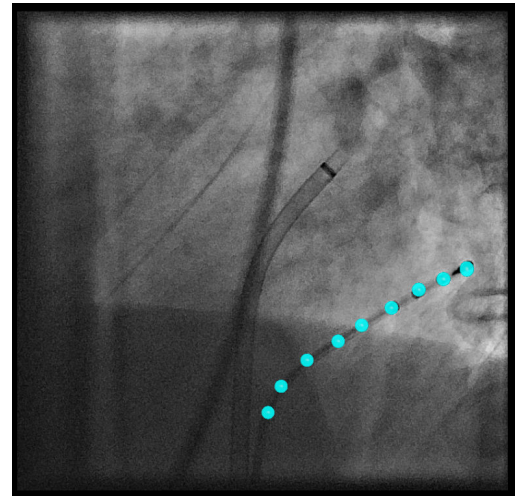

(b) Imaging Plane B

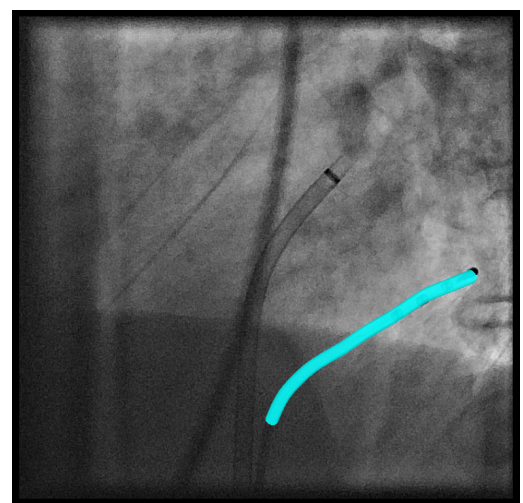

(d) Imaging Plane B

Figure 5: Successive localization by triangulation of the mapping catheter in the coronary sinus from two views.

with $\mathcal{P}_{0}=\mathcal{P}$ using the recent found translation $\mathbf{t}_{k}$. The mean square error for every step during the iteration can be calculated by

$$
d\left(\mathbf{t}_{k}\right)=\frac{1}{N} \sum_{i}\left\|\min _{q_{j} \in \mathcal{Q}}\left(p_{i, k}+t_{k}, q_{j}\right)\right\|^{2} .
$$

We stop our iteration and retrieve the best translation $\hat{\mathbf{t}}$ if either the mean square error does not change significantly any more

$$
d\left(\mathbf{t}_{k+1}\right)-d\left(\mathbf{t}_{k}\right)<10^{-5}
$$

or the number of iterations exceeds a maximum value

$$
k>2000 \text {. }
$$

The translation vector $\hat{\mathbf{t}}$ is then used to shift the mesh of the left atrium in 3-D with the manual segmented coronary sinus to match best to the coronary sinus obtained from the fluoroscopic images.

\section{EVALUATION AND RESULTS}

For evaluation, we considered only translation for registration. Our method was evaluated on eight different data sets. For each of the data sets, a manual registration - considering only 


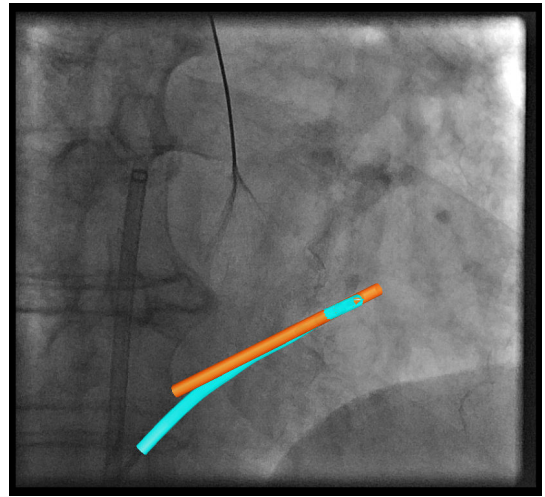

(a) Imaging Plane A

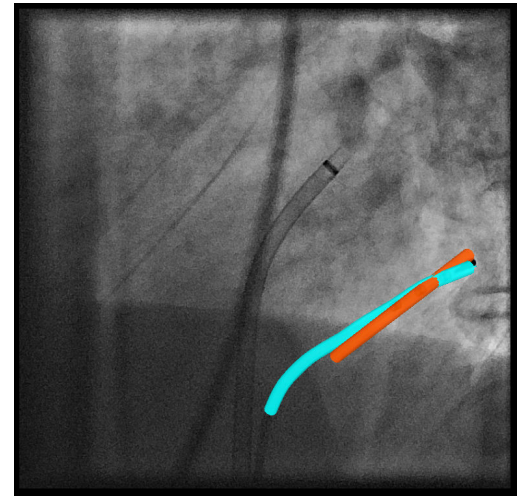

(b) Imaging Plane B

Figure 6: Registration of the extracted coronary sinus (orange) to the mapping catheter placed inside the coronary sinus (blue).

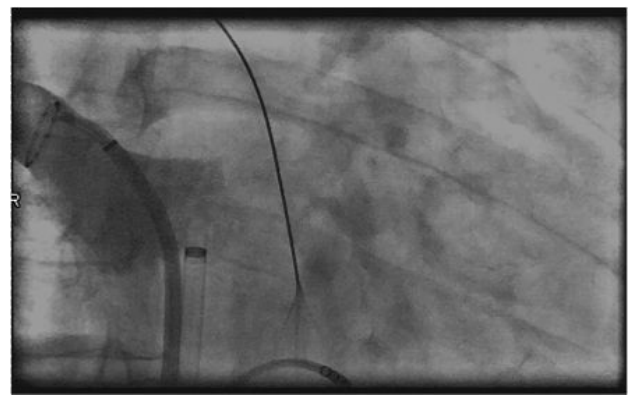

(a)

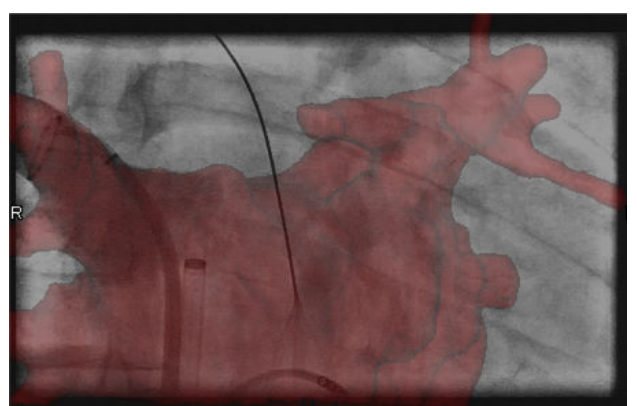

(b)

Figure 7: (a) Contrast injection at the right superior pulmonary vein. (b) MRI overlay registered to contrast injection.

translation as well - had been performed by a physician, denoted as $\mathbf{t}_{\text {phy }} \in \mathbb{R}^{3}$. An example for such a registration is given in Figure 6. We consider these registrations as our gold-standard for further evaluation. To evaluate our ICP approach, we calculate the Euclidean distance between the gold-standard registration and the result obtained by ICP as

$$
\Delta_{\text {reg }}=\left\|\mathbf{t}_{\text {phy }}-\hat{\mathbf{t}}\right\|_{2} \text {. }
$$

Averaged over all data sets, we achieve an average registration error of $5.7 \mathrm{~mm}$ as presented in Figure 8 . This seems to be a large error considering a registration performed by ICP. It has to be noted, that during insertion of the mapping catheter into the coronary sinus, the shape of this vein is changed. In addition, our gold-standard registration might still incorporate user error. Moreover, only one linear catheter could be used for registration. Verification is only possible after the trans-septal puncture, which also moves the left atrium. On different data sets, the possible movement of the heart during the puncture was investigated and we found that on average a re-registration of $10.6 \mathrm{~mm}$ had to be performed [8]. After the trans-steptal puncture, contrast agent is administered to visualize the left atrium, see Figure 7 for an example.

\section{DISCUSSION AND CONCLUSIONS}

We present a first semi-automatic method to register pre-procedural MRI to live fluoroscopic images. This facilitates an initial registration of a 3-D overlay generated from MRI to live X-ray images in the initial phase of the procedure before contrast agent can be administered for a more 


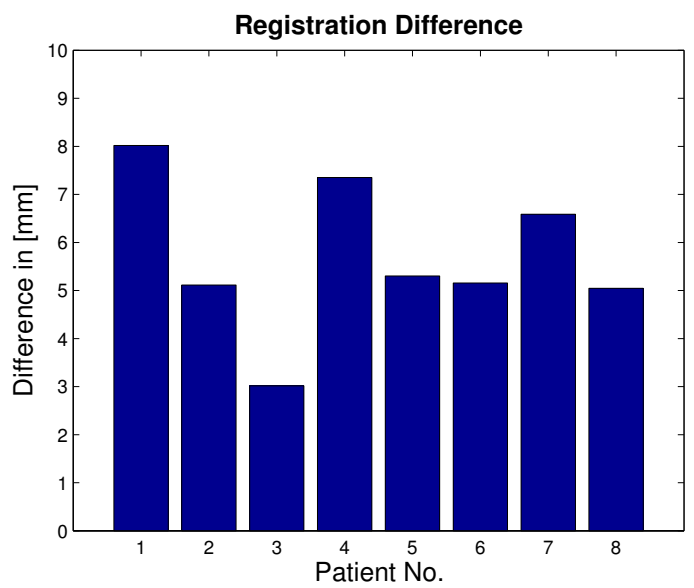

Figure 8: The registration difference $\Delta_{\text {reg }}$ between our gold-standard registration performed by a physician and our ICP approach. The average distance was $5.7 \mathrm{~mm}$.

accurate registration. Our technique provides an early registration method at the beginning of an RFCA procedure for atrial fibrillation. This can be especially interesting for the trans-septal puncture. The use of MR overlay images reduces significantly the radiation exposure for the patient as compared to CT imaging or rotational angiography. Keeping a good registration during the procedure can be facilitated by motion compensation, as shown in $[9,10]$. Extending this method might open the possibility to further reduce the use of contrast agent during the procedure.

\section{Acknowledgments}

The authors would like to thank Marion Lottner and Daniela Mlinaric for their support during the clinical trials. This work has been supported by the German Federal Ministry of Education and Research (BMBF), project grant No. 01EX1012E, in the context of the initiative Spitzencluster Medical Valley - Europäische Metropolregion Nürnberg. Additional funding was provided by Siemens AG, Healthcare Sector.

\section{References}

[1] Wazni, O., Marrouche, N., Martin, D., Verma, A., Bhargava, M., et al., "Radiofrequency ablation vs antiarrhythmic drugs as first-line treatment of symptomatic atrial fibrillation: a randomized trial," JAMA 293, 2634-2640 (2005).

[2] Calkins, H., Brugada, J., et al., "HRS/EHRA/ECAS Expert Consensus Statement on Catheter and Surgical Ablation of Atrial Fibrillation: Recommendations for Personnel, Policy, Procedures and Follow-Up," Heart Rhythm 4, 1-46 (2007).

[3] Ector, J., De Buck, S., Huybrechts, W., Nuyens, D., et al., "Biplane three-dimensional augmented fluoroscopy as single navigation tool for ablation of atrial fibrillation: Accuracy and clinical value," Heart Rhythm 5, 957-964 (2008).

[4] A. Brost, N. Strobel, L. Yatziv, W. Gilson, B. Meyer, J. Hornegger, J. Lewin, and F. Wacker, "Geometric Accuracy of 3-D X-Ray Image-Based Localization from Two CArm Views," in Workshop on Geometric Accuracy In Image Guided Interventions - Medical Image Computing and Computer Assisted Interventions 2009, L. Joskowicz, P. Abolmaesumi, and M. Fitzpatrick, Eds., 12-19, MICCAI, (London UK) (2009).

[5] A. Brost, N. Strobel, L. Yatziv, W. Gilson, B. Meyer, J. Hornegger, J. Lewin, and F. Wacker, "Accuracy of x-ray image-based 3D localization from two C-arm views: a 
comparison between an ideal system and a real device," in Medical Imaging 2009: Visualization, Image-Guided Procedures, and Modeling, M. I. Miga and K. H. Wong, Eds., 7261, 72611Z, SPIE, (Lake Buena Vista, FL, USA) (2009).

[6] P. Besl and N. McKay, "A Method for Registration of 3-D Shapes," IEEE Transactions on Pattern Analysis and Machine Intelligence 14, 239-256 (1992).

[7] S. Rusinkiewicz and M. Levoy, "Efficient Variants of the ICP Algorithm," in Third International Conference on 3D Digital Imaging and Modeling (3DIM 2001), (2001).

[8] F. Bourier, A. Brost, L. Yatziv, J. Hornegger, N. Strobel, and K. Kurzidim, "Coronary Sinus Extraction for Multimodality Registration to guide Transseptal Puncture," in 8th Interventional MRI Symposium, (2010).

[9] A. Brost, R. Liao, N. Strobel, and J. Hornegger, "Respiratory motion compensation by model-based catheter tracking during EP procedures," Medical Image Analysis 14(5), 695 - 706 (2010). Special Issue on the 12th International Conference on Medical Image Computing and Computer-Assisted Intervention (MICCAI) 2009.

[10] A. Brost, A. Wimmer, R. Liao, J. Hornegger, and N. Strobel, "Catheter Tracking: FilterBased vs. Learning-Based," in Pattern Recognition, M. Goesele, S. Roth, A. Kuijper, B. Schiele, and K. Schindler, Eds., Lecture Notes in Computer Science 6376, 293-302, Springer Berlin / Heidelberg (2010). 